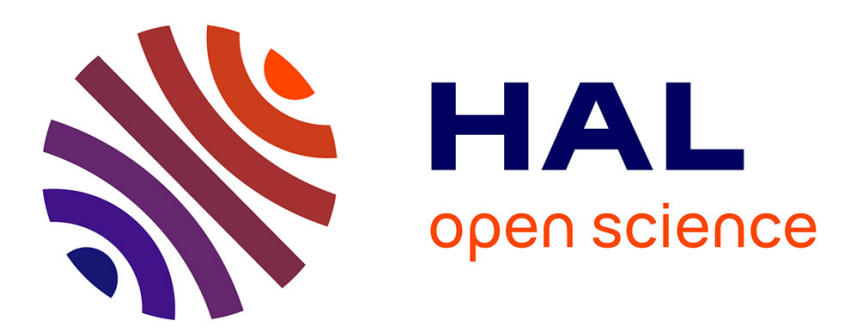

\title{
Inter-relations entre aiguilles de calcite et hyphes mycéliens
}

\author{
Gabriel Callot, André Guyon, Daniel Mousain
}

\section{To cite this version:}

Gabriel Callot, André Guyon, Daniel Mousain. Inter-relations entre aiguilles de calcite et hyphes mycéliens. Agronomie, 1985, 5 (3), pp.209-216. hal-00884752

\section{HAL Id: hal-00884752 \\ https://hal.science/hal-00884752}

Submitted on 1 Jan 1985

HAL is a multi-disciplinary open access archive for the deposit and dissemination of scientific research documents, whether they are published or not. The documents may come from teaching and research institutions in France or abroad, or from public or private research centers.
L'archive ouverte pluridisciplinaire HAL, est destinée au dépôt et à la diffusion de documents scientifiques de niveau recherche, publiés ou non, émanant des établissements d'enseignement et de recherche français ou étrangers, des laboratoires publics ou privés. 


\title{
Inter-relations entre aiguilles de calcite et hyphes mycéliens
}

Gabriel CALLOT, André GUYON \& Daniel MOUSAIN (*)

I.N.R.A., Laboratoire de Science du Sol

(*) Laboratoire de Symbiotes des Racines, Place Viala, F 34060 Montpellier Cedex

RÉSUMÉ

\begin{abstract}
A partir de l'étude des microstructures du sol en place, à l'interface sol-système mycélien, les auteurs mettent en évidence la présence de concentrations calcaires à l'intérieur des hyphes de Basidiomycètes. Ces concentrations minérales s'identifient aux aiguilles de calcite, fréquemment observées dans les sols. L'étude de ces structures minérales très fragiles, rappelle la nécessité d'utiliser des techniques d'observation non destructives (loupe binoculaire) avant d'aborder toutes autres méthodes d'analyses microscopiques.
\end{abstract}

Mots clés additionnels : Microstructure du sol, mycorhizes.

Relation between calcite needles and fungal hyphae in the soil.

Study of soil microstructure at the soil/mycelium interface has shown that calcite accumulations form within the hyphae of Basidiomycetes. These mineral concentrations are identical with the calcite needles which are frequently observed in soil. Non-destructive techniques (binocular microscope) are required to study these very fragile biological and mineral structures before any other microscopic or ultramicroscopic technique is applied.

Additional key words : Microscopic soil fabric, mycorrhizae.

\section{INTRODUCTION}

La description et l'interprétation du mode de formation des aiguilles de calcite rencontrées dans les milieux supergènes (sols ou roches altérées en milieu non saturé) ont toujours suscité un vif intérêt pour les minéralogistes.

Ces aiguilles très fines sont considérées comme de véritables espèces minérales (lublinite), appelées «wiskers " par certains chercheurs spécialistes des karsts. Elles sont, en fait, des concentrations calcitiques et non aragonitiques, à caractère monocristallin (VERGES, 1982). Elles sont décrites en microscopie des sols comme des structures hyphanitiques appartenant au plasma du sol, par suite de leur très faible dimension (diamètre de $0,1 \mu \mathrm{m}$ à $1 \mu \mathrm{m}$ ) (BAL, 1975). Très abondantes dans les encroûtements calcaires (DURAND, 1978 ; POUGET, 1980 ; REGAYA, 1983), elles sont souvent associées à des structures racinaires en cours de minéralogenèse (CALLOT et al., 1983).

L'ensemble des mécanismes de croissance cristalline évoqués jusqu'alors ne fait pas intervenir de processus biologiques, bien que les structures décrites se réfèrent pour la plupart à des qualificatifs à consonance bjologique comme pseudomycélium, structure hyphanitique, croissance dendritique, etc...

De nombreux auteurs ont décrit ces aiguilles de calcite dans des milieux carbonatés non seulement sous climats tempérés, mais aussi dans les milieux tropicaux contrastés (BOCQUIER, 1973 ; NAHON, 1976) dans les sols des zones à climat aride (SEHGAL \& STOOPS, 1972) et dans les sols très organiques où l'activité biologique est intense.

Pour bien mettre en évidence les interactions entre structures biologiques et minérales, nous insisterons sur la nécessité d'observer des échantillons de sol frais, non perturbés, à l'aide d'une loupe binoculaire, avant d'utiliser toute autre technique d'analyse microscopique (lame mince de sol, surfaces polies ou microscopie électronique à balayage). L'analyse structurale (microdissection) sous loupe binoculaire permet de mieux observer les surfaces des agrégats naturels et l'activité des systèmes mycéliens. 


\section{MÉTHODES D'ÉTUDE, PROBLÈME D'ÉCHANTILLONNAGE}

Les diverses concentrations calcaires, en forme d'aiguille, observées dans les sols ou les fissures de roches, à l'aide des techniques habituelles de lames minces de sols ou de roche, ou même de microscopie électronique, n'apparaissent pas souvent en relation directe avec les structures biologiques du système racinaire. Ces absences apparentes de relation, entre structures biologiques et structures minérales proviennent, semble-t-il, d'une insuffisance de précision de l'analyse microscopique habituellement pratiquée en Science du sol ou en Géologie. En effet, les techniques habituelles d'étude des lames minces de sols ou de roches s'adressent à la section d'un volume (analyse d'une surface en 2 dimensions, sur une épaisseur de 10 à $30 \mu \mathrm{m}$ en moyenne). Au cours du séchage et de l'inclusion, les structures biologiques sont souvent détruites ou séparées des structures minérales. En lame mince, la section d'hyphes mycéliens de quelques $\mu \mathrm{m}$ de diamètre est délicate à observer sans technique spéciale de coloration (JONES \& GRIFFITHS, 1964).

Nous avons donc abordé l'analyse microstructurale

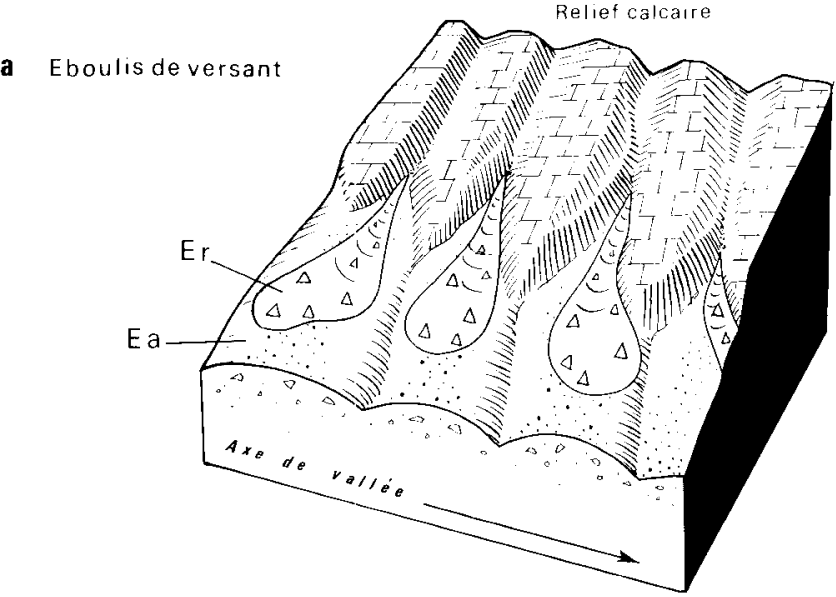

Figure 1

Origine des échantillons :

a) Eboulis de versant, sur calcaires compacts bioclastiques du Crétacé (Alpes du Sud). Ea-éboulis ancien, Er-éboulis récent.

b) Coupe dans cône d'éboulis récent avec cailloutis calcaire non consolidé, très macroporeux.

Hor. A 1-Lit de " platin".

2 - Gravillons roulottés.

Hor. B 3 - Sable limoneux gris-beige très poreux avec gravillons.

Hor. $C_{1 C a} 4-$ Gros éléments anguleux, faiblement encroûtés.

Hor. $\mathrm{C}_{2 \mathrm{Ca}} 5$ - Alternance de lits de graviers isodiamétriques et galets.

Echantillonnage dans zone centrale (cf. Cliché a-Planche I).

$R$ - Racine. du sol en 3 dimensions, observant les structures naturelles du sol à l'interface sol/système racinaire/systèmes mycéliens sur échantillons frais.

Pour s'assurer d'une parfaite observation des structures biologiques et minérales très fragiles, il est indispensable d'observer directement sur le terrain à l'aide d'une loupe binoculaire, ou de prélever des blocs structuraux de dimensions suffisantes, puis de les conserver frais et de procéder à une " dissection » des structures naturelles en laboratoire sous loupe binoculaire (microchirurgie du sol).

\section{Matériaux d'étude}

Les zones à fortes concentrations d'aiguilles de calcite s'observent souvent dans des dépôts de pente caillouteux, calcaires, très poreux, du type éboulis ou « grèzes » (fig. 1).

Dans la partie centrale d'un cône d'éboulis récent où les eaux carbonatées se concentrent, nous avons observé diverses concentrations de calcaire blanchâtre, cotonneux, pulvérulent, à la face inférieure des galets (fig. $1 b$ et cliché a de la planche I).

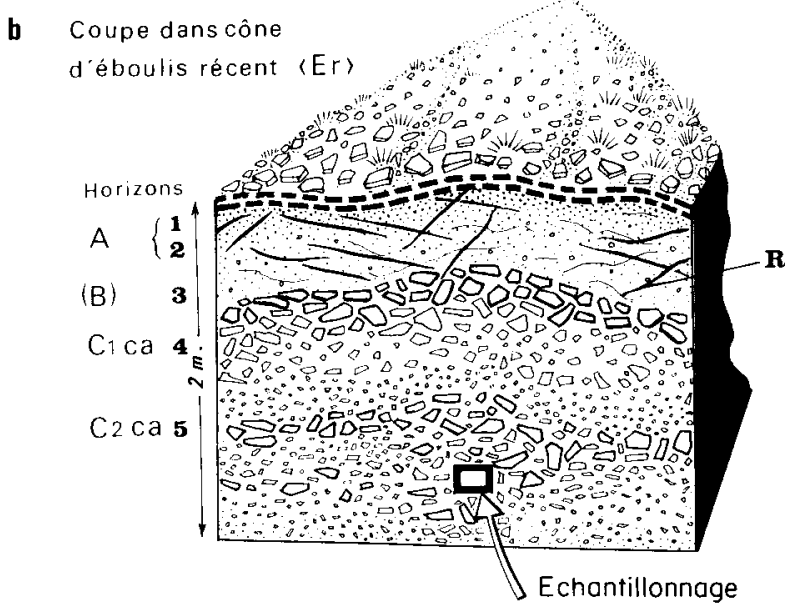

Origin of samples :

a) Rock fall ( $E a$ - ancient, Er - recent) on hard bioclastic Cretaceous limestone in the Southern Alps.

b) Profile of a recent rock fall, with non-cemented, highly macroporous calcareous stones.

Hor. A 1 - Flat pebble horizon.

2 - Rounded graved.

Hor. B 3 - Highly porous grey silt with gravel.

Hor. $C_{l \mathrm{Ca}} 4$ - Large sharp fragments, weakly cemented.

Hor. $C_{2 \mathrm{Ca}} 5$-Alternation of beds of isodiametric gravel and pebbles.

Sample in central zone (cf. fig. a-Plate I).

$R-R o o t$.
Planche I

Aiguilles de calcite dans un encroûtement calcaire mycélien.

Coupe aa : Croquis d'après lame mince de l'encroûtement calcaire développé à la face inférieure d'un caillou du cliché $a$.

1 - Structure de roche,

2 - Liseré brunâtre d'interface,

3 - Sphérules radiaires de calcite sparitique,

4 - Plasma micritique complexe,

5 - Réseau dense d'aiguilles de calcite.

Clichés :

a - Macrophotographie/terrain montrant le développement d'un voile blanchâtre, calcaire $(L)$ à la face inférieure des cailloux.

$b$ - Sphérules de calcite jaunâtre (S) (structure 3 de coupe aa).

$c$ - Cloisonnement blanchâtre construit par les micro-arthropodes (M) (structure 5 de coupe aa). $c_{1}$ - Détail d'une cloison $(M)$ en microscopie électronique montrant les aiguilles de calcite cassées; (L) aiguilles dans les vides.

$d$ - Voile blanchâtre d'aiguilles de calcite ( $L)$ dans les macrovides avec hyphes et cordons mycéliens (Cm).

$d_{I}$ - Détail montrant les aiguilles de calcite (L) autour d'un cordon mycélien $(\mathrm{Cm})$.

Plate I

Needle shaped calcite in a " mycelial 》 calcareous cimented horizon. Fig aa : Fine section of a calcareous accumulation on the lower surface of a pebble (after fig. a).

1 - Rock structure.

2 - Brown interface line. 

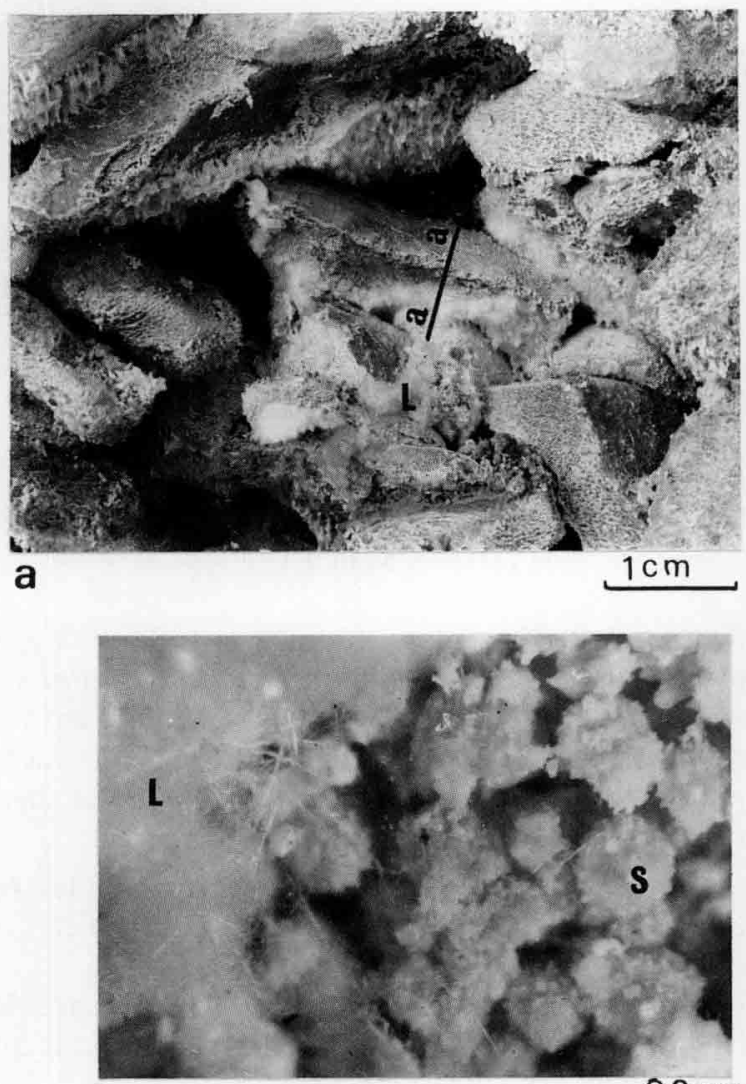

\section{b Détail de 3}

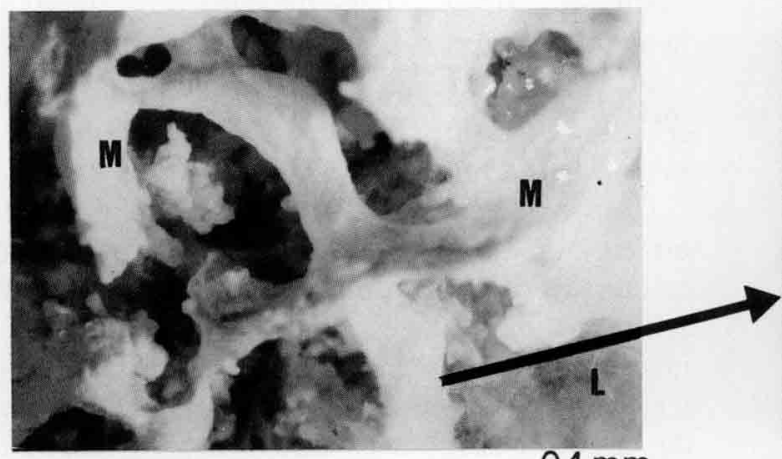

C Détail ae 5

$0,4 \mathrm{~mm}$

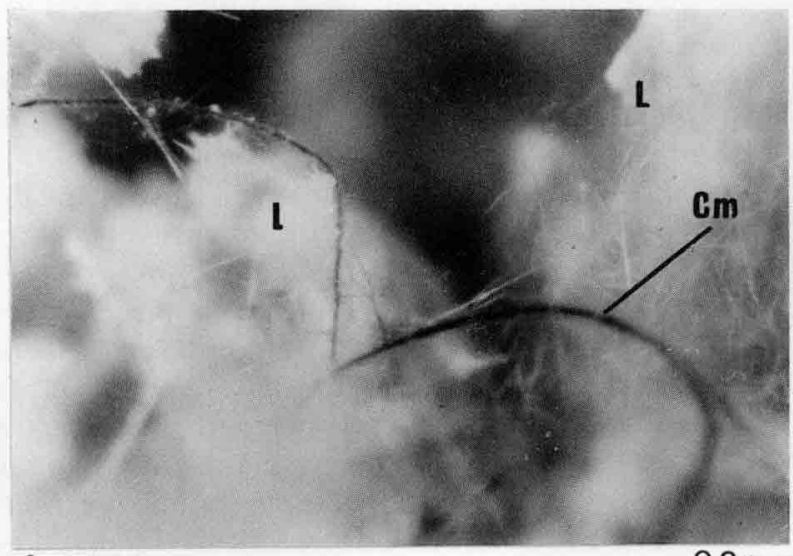

d Aiguilles de calcite

$\underline{0,2 \mathrm{~mm}}$

3 - Radial spheroids of sparitic calcite.

4 - Complex micritic plasma fabric.

5 - Hyphanitic plasma fabric of calcite needles.

Fig :

$a$ - Field photograph showing the white calcareous veil $(L)$ on lower surface of a calcareous pebble.

$b-$ Yellow calcite spheroids (Detail 3 of fig. aa).

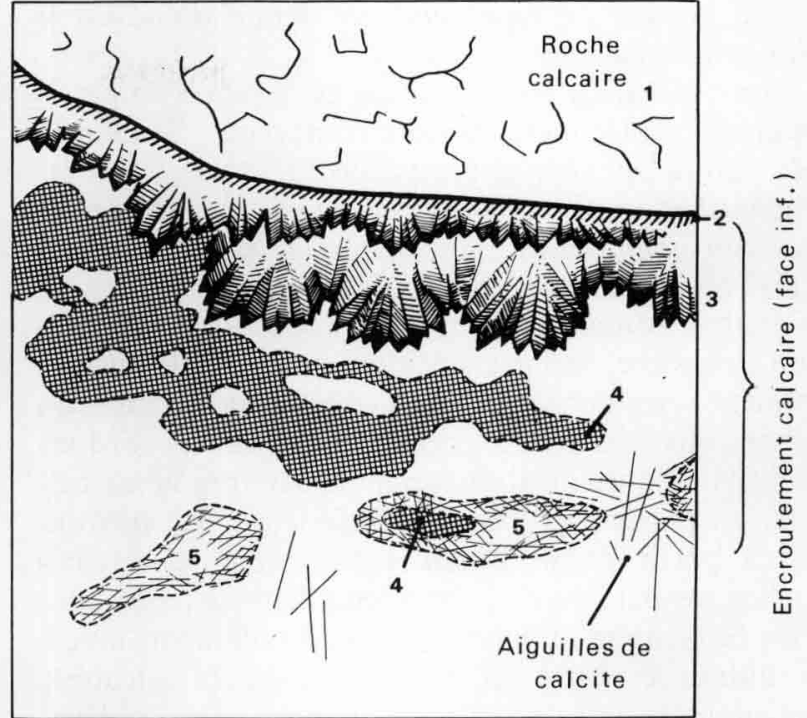

$100 \mu \mathrm{m}$ COUPE a a sur photo $\mathbf{a}$
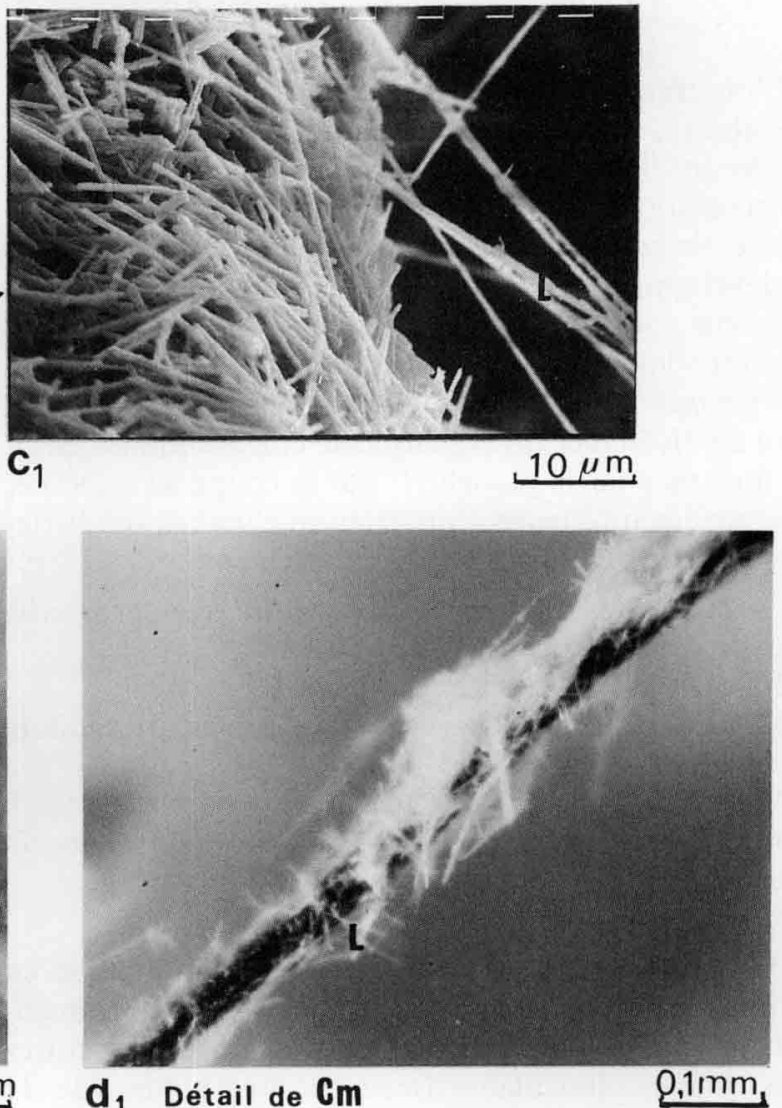

d Détail de $\mathbf{C m}$ $c_{l}$ - Detail of partition (M) with broken calcite needles scanning electron microscopy; calcite needles $(L)$ in the cavities.

$d-$ White veil of calcite needles $(L)$ in macro-cavities with hyphae and mycelial strands $(\mathrm{Cm})$.

$d_{l}-$ Detail of mycelial strand $(\mathrm{Cm})$ with calcite needles $(L)$. 
- Le sol est de type rendzine brune d'éboulis à encroûtement calcaire.

- La végétation très dispersée est typique de garrigues méditerranéennes. La flore comprend : Staehlinia dubia, Santolina chamaecyparissus, Thymus vulgaris, Aphyllanthes monspeliensis et quelques arbustes: Pirus amygdaliformis, Sorbus aria et pins noirs d'Autriche (Pinus nigra nigricans).

L'analyse sommaire du système racinaire de la végétation arbustive, montre un intense réseau de racines fortement mycorhizées dans les couches supérieures du sol (horizons A et B). Les petites racines et les cordons mycéliens colonisent abondamment les fragments calcaires, en particulier leur face inférieure. En profondeur, à partir de l'horizon 4 (fig. $1 b$ ), les racines actuelles ne pénètrent qu'accidentellement ; par contre, on peut suivre un intense réseau de filaments mycéliens autour et entre les cailloux et galets calcaires. Enfin, dans le niveau 5, l'encroûtement calcaire développé à la face inférieure des cailloux est constitué par un voile blanchâtre calcaire, très fragile, à travers lequel il est possible, par une simple observation macroscopique, de reconnaître les traces d'activité biologique et les cordons mycéliens.

C'est dans ce niveau que nous avons réalisé l'analyse microstructurale.

\section{DISTRIBUTION MICROSTRUCTURALE DES RÉSEAUX D'AIGUILLES}

L'observation de l'encroûtement calcaire et du voile blanchâtre, effectuée d'abord sous loupe binoculaire, sur échantillons frais non perturbés, a montré de fortes concentrations d'aiguilles de calcite, souvent anastomosées en cordons ; certaines aiguilles isolées très fines et développées dans les vides, peuvent atteindre une longueur de l'ordre de $0,5 \mathrm{~cm}$ : soit un rapport diamètre/longueur de l'ordre de $1 / 10^{4}$ ! L'analyse en lame mince de la face inférieure des caillowx permet ainsi de distinguer un ensemble de constructions calcaires illustrées sur la planche I. Sur la coupe aa effectuée à la surface inférieure d'un galet du cliché a, on distingue à partir de la base de l'encroûtement :

1 - Une structure de roche calcaire avec gros cristaux de calcite (sparite) et bioclastes.

2 - Un liséré brunâtre d'interface.

3 - Diverses couches de bourgeonnement radiaire de calcite.

4 - Un plasma micritique calcaire complexe.

5 - Divers réseaux d'aiguilles de calcite enchevêtrées.

6 - Des aiguilles de calcite isolées.

Pour donner à cette description pétrographique en 2 dimensions une valeur spatiale (analyse en 3 dimensions), nous avons identifié les principales structures sous loupe binoculaire (macrophotographies de la planche I), avant imprégnation de l'échantillon.

L'étude des échantillons sous loupe binoculaire nous a permis :

- de préciser les structures radiaires (3) qui apparaissent sous forme de sphérules jaunâtres (cliché b) probablement d'origine alguaire ;

- de mettre en évidence un réseau de cloisons blan- châtres (cliché c) construit par les micro-arthropodes avec des aiguilles de calcite cassées (cliché $c_{1}$ );

- enfin, de constater que les aiguilles de calcite isolées de la coupe aa, sont étroitement associées aux cordons et filaments mycéliens $(\mathrm{cm})$ qui sillonnent l'ensemble de l'encroûtement (cliché d).

Le voile blanchâtre cotonneux (clichés $\mathrm{d}, \mathrm{d}_{1}$ ), constitué par des aiguilles de calcite mêlées à des hyphes mycéliens blancs ou bruns, peut atteindre une épaisseur de 1 à $3 \mathrm{~cm}$. Il se développe toujours à la face inférieure des galets et cailloux dans les macropores.

\section{RELATIONS AIGUILLES DE CALCITE - HYPHES MYCÉLIENS}

Pour préciser les relations entre aiguilles et hyphes mycéliens, nous avons isolé par microdissection sous loupe binoculaire, les cordons mycéliens entourés d'aiguilles (cliché $\mathrm{d}_{1}$ de planche $\mathrm{I}$ ). L'étude en microscopie électronique à balayage a permis ainsi de distinguer en particulier (planche II) :

- des filaments cylindriques agrégés, rigides (clichés a et $\mathrm{a}_{1}$ ) avec boucles dangeardiennes (bd) caractéristiques des Basidiomycètes;

- un réseau d'aiguilles de calcite (L), soit accolées entre elles (clichés a et b) ou aux hyphes cylindriques rigides (cliché $\mathrm{a}_{1}$ ), soit isolées (clichées $\mathrm{b}, \mathrm{c}, \mathrm{c}_{1}$ ) ;

- des concentrations dentelées de calcite (cc) autour desquelles subsistent les parois $\mathrm{P}$ des filaments mycéliens (clichés $\mathrm{c}$ et $\mathrm{c}_{1}$ ).

Cette analyse microstructurale fait apparaître que ces concentrations calcitiques se produisent à l'intérieur des filaments mycéliens.

Contrairement aux structures cellulaires des racines où chaque cellule est délimitée par une paroi pectocellulosique, la communication entre les différentes cellules d'un hyphe est assurée par des cloisons souvent incomplètes. Cette structure facilite les échanges entre les cellules, et peut alors expliquer la croissance rapide de ces concentrations calcitiques intracellulaires, en forme d'aiguille. Il est à noter que la forme dentelée des aiguilles, préexiste déjà à l'intérieur de l'hyphe (cliché $\mathrm{c}_{1}$, planche II).

La présence d'hyphes de champignons associés aux réseaux d'aiguilles de calcite a été confirmée par la mise en évidence de chitine associée à la calcite (mesure colorimétrique après hydrolyse acide, selon PLASSARD et al., 1982), la chitine étant un élément caractéristique des parois des hyphes de champignons.

Dans certains cas, les concentrations en carbonate de calcium peuvent s'effectuer dans les parois ce qui explique l'existence de baguettes calcitiques creuses et lisses. Enfin, la présence de tenons d'anastomose calcitisés (E), conservés sur certaines aiguilles de calcite (cliché b de planche III), montre l'étroite relation qui existe entre hyphes et aiguilles de calcite. Les tenons d'anastomose sont des éléments qui relient certains hyphes individuels à l'intérieur d'un cordon (cf. cliché $b_{1}$ de la planche II).

Dans les études sur la calcitisation des filaments mycéliens, les auteurs font intervenir des phénomènes de croissance cristalline à partir des parois (à l'intérieur ou à l'extérieur), après la mort du filament mycélien (KOBLUK \& RISK, 1977 ; KLAPPA, 1979). La présence 

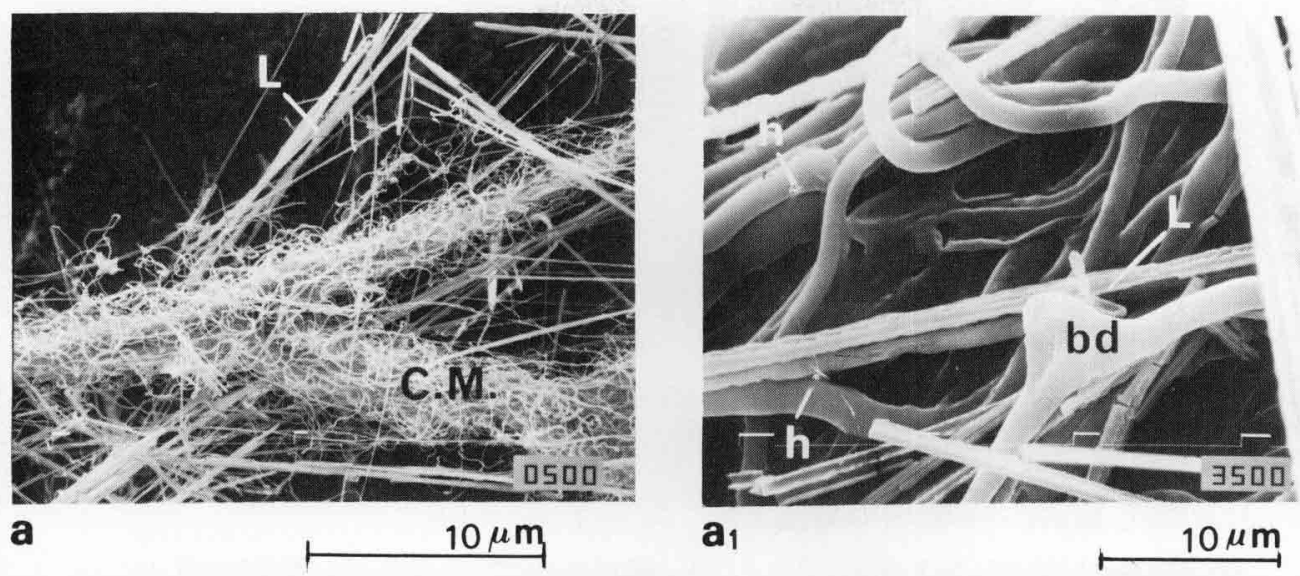
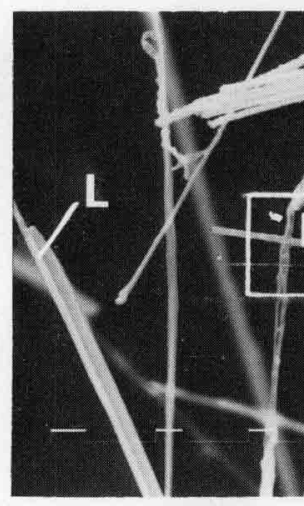

b

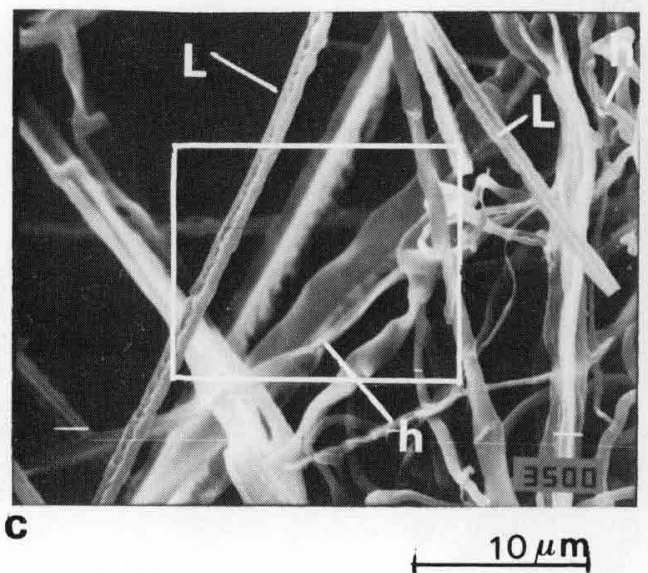

Planche II

Relations entre aiguilles de calcite et hyphes mycéliens.

(Analyse en microscopie électronique d'un voile mycélien, échantillon extrait du cliché d de la planche I.)

Clichés :

a-Cordon mycélien (CM) constitué par un ensemble d'hyphes cylindriques et d'aiguilles de calcite ( $L$ ).

$a_{I}$ - Détail des hyphes ( $h$ ) avec boucle dangeardienne (bd) et aiguille de calcite ( $L$ ).

b - Réseau d'hyphes rigides $(H)$ et aiguilles de calcite $(L)$.

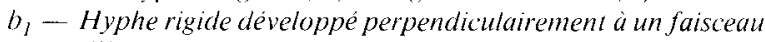
d'hyphes anastomosés: boucle dangeardienne (bd), tenons d'anastomose (E).

$c-$ Hyphes souples et aplatis (h) avec aiguilles de calcite dentelées $(L)$

$c_{I}$ - Détail de coù l'on observe au contact d'une enveloppe organique (parois $P$ de l'hyphe) des concentrations dentelées de calcite (cc) comparables aux aiguilles $(L)$.
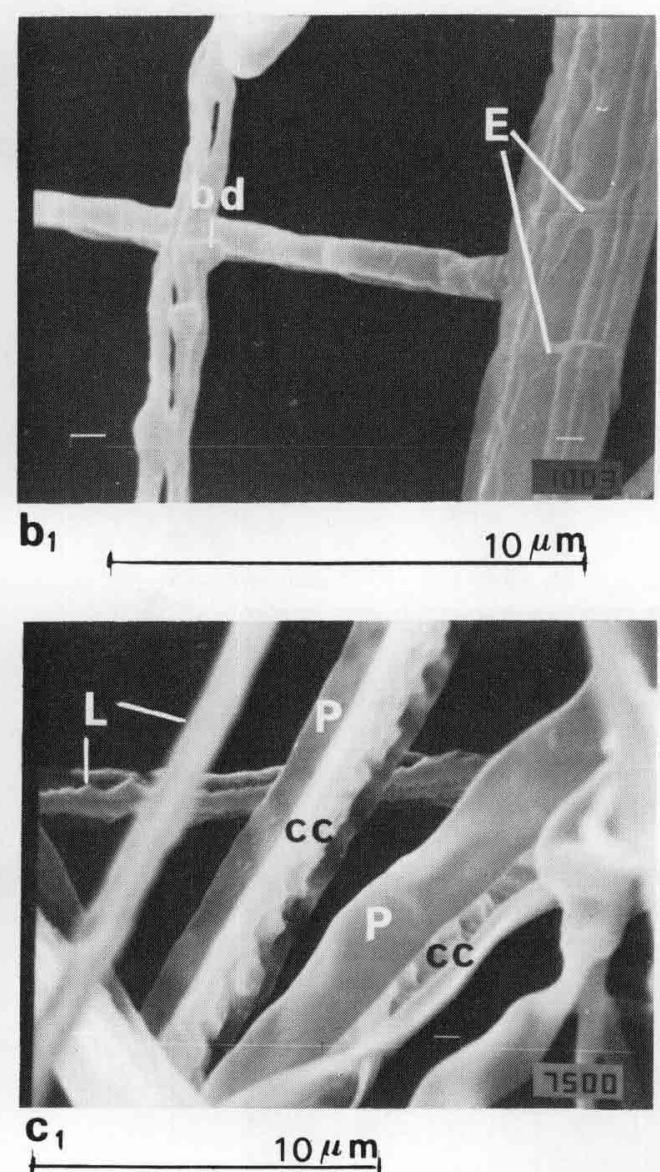

Plate II

Relation between needle shaped calcite and hyphae.

(Scanning electron micrograph of sample from d, plate 1.) Fig :

$a-$ Mycelial strand (CM) organized with cylindrical hyphae and calcite needles $(L)$.

$a_{1}$ - Detail of a Basidiomycete hypha (h) with clamp connection (bd) and calcite needle $(L)$.

$b-$ Network of rigid hyphae $(H)$ and calcite needles $(L)$.

$b_{1}$ - Rigid hypha perpendicular to a sirand of anastomosing hyphae ; clamp connection (bd), anastomosis (E).

$c$ - Flexible flattened hyphae (h) with toothed calcite needles (L.). $c_{1}-$ Detail of $c$ showing, in contact with the organic surface (hyphal wall P), toothed concentrations of calcite (c) similar to needles (L.). 


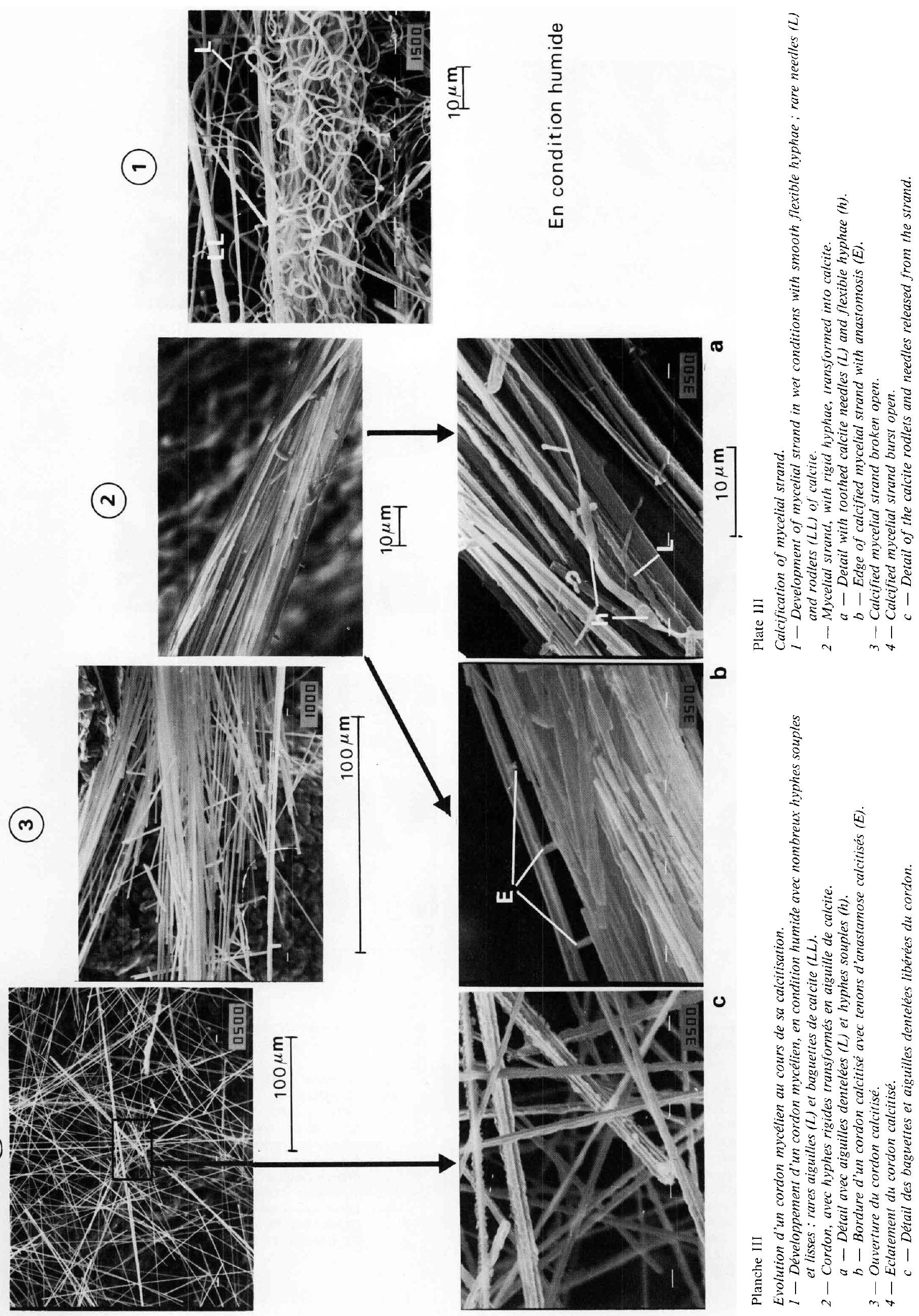


de calcite à l'intérieur de l'hyphe, semblerait montrer que les phénomènes initiaux de croissance cristalline de ces aiguilles peuvent se produire lorsque l'organisme est encore vivant. La rapidité de formation et de dissolution de ces structures cristallines distingue, semble-til, ce processus de minéralogenèse sous influence biologique de la fossilisation des filaments calcifiés conservés dans les structures sédimentaires.

\section{MICROANALYSE COMPARÉE DES HYPHES MYCÉLIENS ET AIGUILLES DE CALCITE}

L'analyse comparée des teneurs en calcium et silicium des hyphes mycéliens et de différentes sortes d'aiguilles a été faite à la microsonde, grâce à l'aimable collaboration de $\mathrm{Cl}$. MERLET (Service commun «Microsonde », U.S.T.L. Montpellier). Dans le cas de l'hyphe conducteur, hyphe cylindrique suffisamment rigide, d'un diamètre de $8 \mu \mathrm{m}$, nous avons relevé des teneurs notables en silicium $(0,67$ p. 100 du poids de matière sèche) et en potassium, avec absence de calcium, d'aluminium ou de phosphore décelable en microanalyse (fig. 2).

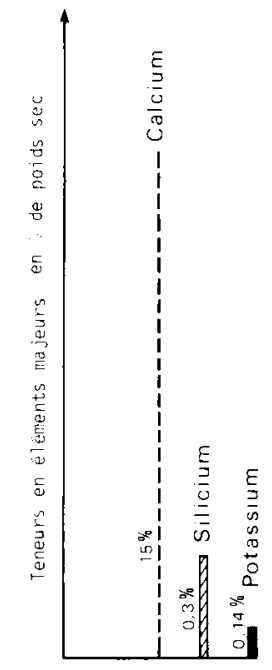

Echelle relative

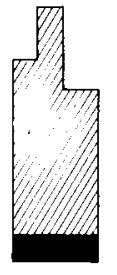

Hyphe

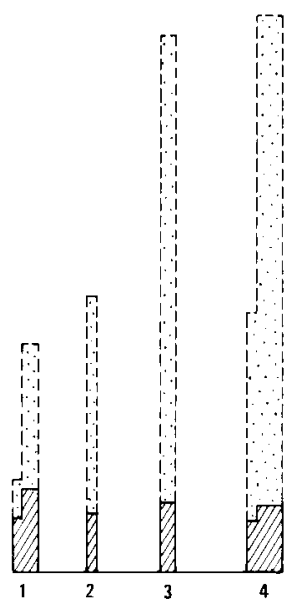

Aiguilles decalcite
1 - lisse et ronde ;

2 - fine, légèrement dentelée :

3 - légèrement dentelée avec parois de l'hyphe

4 - lisse, anastomosée (bâtonnet).

Figure 2

Histogramme des teneurs comparatives en calcium et silicium dans les hyphes mycéliens et aiguilles de calcite (d'après analyse à la microsonde type CAMEBAX).

Figure 2

Calcium and silicon content of mycelial hyphae and calcite needles (after microprobe analysis, CAMEBAX).

Dans les différents types d'aiguilles, on peut noter une certaine constance des teneurs en silice, de l'ordre de 0,3 p. 100 de la matière sèche. Les concentrations en calcium, variables selon les types d'aiguilles, évoluent entre 5 et 15 p. 100 de la matière sèche. Dans les échantillons analysés, le calcium présent sous forme de carbonate de calcium correspondait à 10 à 40 p. 100 de la masse totale. L'analyse par diffraction $X$ des aiguilles a confirmé la présence effective de calcite et non d'aragonite.

Les échantillons analysés dans ces structures mycéliennes présentent des concentrations en carbonate de calcium moins élevées que celles données par BUTEL (1982) dans les sols du Poitou. Il semble possible qu'il puisse se produire une évolution cristalline de ces aiguilles postérieurement à leur formation intracellulaire. La diminution des teneurs en silicium dans les aiguilles de calcite $(0,20 \mathrm{p} .100)$ comparativement à celles relevées dans un hyphe non calcitisé $(0,67$ p. 100) pourrait s'expliquer par la solubilisation de la silice au cours de la précipitation du carbonate de calcium.

Dans ces aiguilles, la présence de silice d'ailleurs soulignée par d'autres auteurs (voir BUTEL, 1982, par exemple), pourrait assurer une certaine rigidité à ces structures cristallines.

\section{CALCITISATION EXPÉRIMENTALE D'UN CORDON MYCÉLIEN}

Des concentrations d'aiguilles sont généralement observées dans des milieux poreux et aérés où l'eau fortement chargée en calcium circule de manière préférentielle. Ces milieux sont aussi très secs en période estivale. Pour simuler ces conditions naturelles en laboratoire, deux cailloux calcaires et leur voile mycélien ont été soumis aux conditions suivantes :

- l'un a été placé dans une atmosphère humide, constamment imbibée d'eau goutte à goutte,

- l'autre a été conservé séché à l'air.

En atmosphère humide, nous avons observé, après quelques jours, le développement de nouveaux hyphes mycéliens. La croissance de certains de ces hyphes semble d'ailleurs s'effectuer à partir des gouttelettes de percolation dans certains microsites. D'autre part, les aiguilles de calcite subissent une transformation progressive, avec des phénomènes de dissolution et reprécipitation. Dans ces conditions humides, les aiguilles sont souples et souvent curvilignes.

Le voile mycélien séché à l'air est devenu par contre très fragile, les aiguilles cassantes. Nous avons pu observer en particulier « l'éclatement » des cordons mycéliens calcitisés (planche III). Cet éclatement aboutit progressivement à la libération des aiguilles de calcite, ainsi séparées de leur structure biologique originelle. Les tenons d'anastomose $(\mathrm{E})$ calcitisés conservés sur certaines aiguilles (cliché b de la planche III) témoignent de leur appartenance originelle à un cordon.

Des études de bio-pédologie expérimentale en conditions contrôlées devraient permettre de préciser le mécanisme de croissance de ces aiguilles et les conditions thermiques et hydriques favorables à leur formation.

\section{CONCLUSION}

Dans l'article précédent (CALLOT et al., 1985), nous avons mis en évidence à partir d'études expérimentales les possibilités de concentration du carbonate de calcium à l'extérieur dés hyphes de champignon. Dans le 
milieu naturel, ces concentrations peuvent également se développer à l'intérieur de l'hyphe. Ce phénomène de concentration du carbonate de calcium par les hyphes mycéliens pourrait être en relation avec la présence de granules de polyphosphate (STRULLU et al., 1981). Ces microsystèmes constituent en fait des pièges à calcium, germes de nucléation des cristaux de carbonate de calcium. Comme nous avons pu l'observer en étude expérimentale, cette calcitisation des structures fongiques est favorisée par le dessèchement, mécanisme qui accélère la concentration des sels. Le dessèchement des hyphes serait, semble-t-il, toujours accéléré lorsque les hyphes se développent dans les vides, sans contact avec un squelette. Ceci pourrait expliquer en partie la présence des aiguilles de calcite dans les macrovides du sol où, par ailleurs, leur élongation n'est nullement freinée par des contraintes physiques.
A travers l'exemple des aiguilles de calcite, structures cristallines très fragiles, nous avons également insisté sur la nécessité d'aborder l'étude des microstructures du sol en 3 dimensions, pour mieux mettre en évidence les relations entre formes biologiques et structures minérales.

Recu le 10 octobre 1983 Accepté le 18 octobre 1984.

\section{REMERCIEMENTS}

Nous tenons à remercier Monsieur J. COUDERC, dessinateur cartographe au Laboratoire de Science du Sol de Montpellier, pour son aide précieuse dans la composition des planches et dessins, ainsi que le Pr. L. SAlsac, Directeur du Laboratoire de Recherches sur les Symbiotes des Racines (I.N.R.A. Montpellier) pour ses fructueuses critiques du manuscrit.

\section{RÉFÉRENCES BIBLIOGRAPHIQUES}

Bal L., 1975. Carbonate in soil : A theorical consideration on and proposal for its fabric analysis. 1. - Crystic, calcic and fibrous plasmic fabric. Neth. J. agric. Sci., 23, 18-35 et 163-176.

Bocquier G., 1973. Genèse et évolution de deux roposéquences de sols tropicaux du Tchad - Interprétation biogéodynamique. Thèse Doct. ès Sci., Parịs, Mém. ORSTOM, 350 p.

Butel P., 1982. Formes et mécanismes de l'accumulation carbonatée dans les sols de la plaine poitevine. Thèse $3^{\mathrm{e}}$ cycle, Univ. Poitiers, $122 \mathrm{p}$.

Callot G., Chamayou H., Maertens C., Salsac L., 1983. Interactions sol/racine et incidence sur la nutrition minérale. I.N.R.A., Paris, $320 \mathrm{p}$.

Callot G., Mousain D., Plassard C., 1985. Concentrations de carbonate de calcium sur les parois des hyphes mycéliens. Agronomie, 5 (2), 14.3-150.

Durand D., 1978. La pédogenèse en pays de craie dans le Nord-Est de la France. Thèse Doct. ès Sci., Institut de Géologie, Strasbourg, $175 \mathrm{p}$.

Jones D., Griffiths El., 1964. The use of thin soil sections for the study of soil micro-organisms. Plant and Soil, 20 (2), 232-240.

Klappa C. F., 1979. Calcified filaments in quaternary calcretes : organo-mineral interactions in the subaerial vadose environnement. J. Sediment Petrol., 49 (3), 955-968.

Kobluk D. R., Risk M. J., 1977. Calcification of exposed filaments of endolithic algae, micrite envelope formation and sediment production. Sediment Petrol., 47 (2), 517-528.

Nahon D., 1976. Cuirasses ferrugineuses et encroûtements calcaires au Sénégal occidental et en Mauritanie. Systèmes évolutifs : géochimie, structures relais et coexistence. Thèse Doct. ès Sci., Fac. Sci. Tech. St-Jérôme, Marseille, Mém. C.N.R.S. 44, 232 p.

Plassard C., Mousain D., Salsac L., 1982. Estimation of mycelial growth of Basidiomycetes by means of chitin determination. Phytochemistry, 21 (2), 345-348.

Pouget M., 1980. Les relations sol/végétation dans les steppes algéroises. Thèse Doct. ès Sci., Paris, Travaux et documents ORSTOM, $116,555 \mathrm{p}$.

Regaya K., 1983. Etude géologique de la formation des limons de Matmata (Sud Tunisien). Thèse $3^{\mathrm{e}}$ cycle, Marseille St-Jérôme, 121 p.

Sehgal J. L., Stoops G., 1972. Pedogenetic calcite accumulation in arid and semi-arid regions of the Indogangetic alluvial plain of erstwhile Punjab (India). Their morphology and origin. Geoderma, 8, 59-72.

Strullu D. G., Gourret J. P., Garrec J. P., 1981. Microanalyse des granules vacuolaires des ectomycorhizes, endomycorhizes et endomycothalles. Physiol. Vég., 19, 367-378.

Verges V., 1982. Contribution à l'analyse et à la représentation cartographique des formations pédologiques en moyenne montagne calcaire. Thèse $3^{\mathrm{e}}$ cycle, Univ. Paris VII, Pedol. Aménagement des Sols, 223 p. 